\title{
Final Focus Challenges for Muon Colliders at Highest Energies
}

\author{
F. Zimmermann \\ CERN, SL Division, Geneva, Switzerland
}

\begin{abstract}
This report discusses challenges in developing final-focus systems for muon colliders at 10-100 TeV [1]. The optics design is impeded by limited quadrupole gradients and the large beam emittances. Of interest are also spot-size dilutions accumulating over several turns. Tolerances on magnet vibration and field stability are comparable to those at future electron-positron linear colliders. While at $10 \mathrm{TeV}$ nonlinear kinematic terms are still important, at $100 \mathrm{TeV}$ synchrotron radiation may complicate the design. In view of the high charge per bunch and the multiple passes, wake fields and space-charge effects must be looked at carefully. For multi-TeV energies a single-pass muon collider is a promising option, since it poses no neutrino radiation hazard, can accommodate ultracold muon beams, and lends itself more easily to novel focusing techniques, such as plasma lenses or dynamic focusing, thereby avoiding the gradient limitations of conventional quadrupoles.*
\end{abstract}

Geneva, Switzerland

January 11, 2000

\footnotetext{
* Presented at HEMC'99 workshop, "Studies on Colliders and Collider Physics at the Highest Energies: Muon Colliders at $10 \mathrm{TeV}$ to $100 \mathrm{TeV}$ ", Montauk, New York, 27 September - 1 October, 1999.
} 


\section{Overview}

Table 1 compares the interaction-point (IP) parameters for a $10-\mathrm{TeV}$ and a $100-$ $\mathrm{TeV}$ muon collider with their counterparts in a few existing or planned $\mathrm{e}^{+} \mathrm{e}^{-}$ linear colliders. The IP beta functions, bunch length, and the interaction-point (IP) transverse spot sizes for a high-energy muon collider are comparable to those successfully obtained at the Stanford Linear Collider (SLC). All these values are quite relaxed compared with the corresponding numbers for a future $\mathrm{e}^{+} \mathrm{e}^{-}$ collider. Moreover, the expected rms energy spread is extremely small, which softens constraints on the final-focus energy bandwidth.

However, four factors could complicate the final-focus design:

- the extremely high beam energy, in the face of limited quadrupole strengths, and large transverse emittances;

- a factor 20-75 higher bunch charge than SLC (factor 200-750 larger than CLIC);

- synchrotron radiation at $100 \mathrm{TeV}$;

- the multiple passes.

In the following sections we will discuss potential problems associated with each of these items.

\section{Optics}

The primary difficulty in designing a final-focus optics is the limited quadrupole strength. Within the reach of present technology is a quadrupole gradient of 320 $\mathrm{T} / \mathrm{m}$ [5]. Even if we optimistically extrapolate this to $500 \mathrm{~T} / \mathrm{m}$, at $100 \mathrm{TeV}$ the corresponding normalized gradient is a meager $k \approx 3 \times 10^{-3} \mathrm{~m}^{-2}$. This implies that the final quadrupole magnets must be long, and that at least in one plane the beta functions can grow to large values, generating substantial chromaticity, which reduces the momentum bandwidth in that plane.

We used the automatic final-focus design program FFADA [6] and the general accelerator design code MAD [7] to develop a series of six test optics. Each of the final-focus models consists of three parts: a horizontal chromatic correction section (CCX), a vertical chromatic correction section (CCY) and a final transformer, usually consisting of two doublets. Each chromatic correction section comprises 4 bending sections and a $-I$ pair of sextupoles, positioned near the maximum dispersion points. The $-I$ transform between the sextupoles ensures that geometric aberrations and second-order dispersion induced by either sextupole cancel, and only chromaticity is generated. Residual aberrations, due to the chromatic breakdown of the $-I$, are of 4 th order or higher. For the purpose of this exercise, the final transformer demagnification factor was chosen as 20 in the horizontal plane and 50 in the vertical, a free length of $2 \mathrm{~m}$ was assumed 
Table 1: Comparison of final-focus and IP parameters for several $\mathrm{e}^{+} \mathrm{e}^{-}$and multi$\mathrm{TeV} \mu^{+} \mu^{-}$colliders: the Stanford Linear Collider (SLC) is the first and so far only operating linear collider [2]; the Next Linear Collider (NLC) is the design for a future 1-TeV $\mathrm{e}^{+} \mathrm{e}^{-}$collider [3]; and CLIC [4] is a multi-TeV linear collider under study at CERN. The numbers for the 10 and 100-TeV muon colliders, $\mu$-A and $\mu-\mathrm{B}$, correspond to the parameters provided by the workshop organizers.

\begin{tabular}{lcccccc}
\hline parameter & symbol & SLC & NLC & CLIC & $\mu$-A & $\mu$-B \\
\hline \hline species & & $\mathrm{e}^{+} \mathrm{e}^{-}$ & $\mathrm{e}^{+} \mathrm{e}^{-}$ & $\mathrm{e}^{+} \mathrm{e}^{-}$ & $\mu^{+} \mu^{-}$ & $\mu^{+} \mu^{-}$ \\
cm energy [TeV] & $E_{c o m}$ & 0.1 & 1 & 3 & 10 & 100 \\
Lorentz factor & $\gamma$ & $10^{5}$ & $10^{6}$ & $3 \times 10^{6}$ & $5 \times 10^{4}$ & $5 \times 10^{5}$ \\
bunch population [1010] & $N_{b}$ & 4 & 1 & 0.4 & 300 & 80 \\
hor. emittance $[\mu \mathrm{m}]$ & $\gamma \epsilon_{x}$ & 50 & 4.5 & 0.68 & 38 & 8.7 \\
vert. emittance $[\mu \mathrm{m}]$ & $\gamma \epsilon_{y}$ & 8 & 0.1 & 0.02 & 38 & 8.7 \\
hor. beta [mm] & $\beta_{x}^{*}$ & 2.8 & 12 & 8 & 2.1 & 2.5 \\
vert. beta [mm] & $\beta_{y}^{*}$ & 1.5 & 0.15 & 0.15 & 2.1 & 2.5 \\
hor. spot size [nm] & $\sigma_{x}^{*}$ & 1700 & 235 & 43 & 1300 & 210 \\
vert. spot size [nm] & $\sigma_{y}^{*}$ & 900 & 4 & 1.0 & 1300 & 210 \\
bunch length [mm] & $\sigma_{z}$ & 1 & 0.12 & 0.03 & 2.2 & 2.5 \\
rms energy spread [\%] & $\sigma_{\delta}$ & 0.1 & 0.3 & 0.3 & 0.06 & 0.011 \\
pinch enhancement & $H_{D}$ & 2.0 & 1.45 & 2.24 & 1.08 & 1.11 \\
\hline
\end{tabular}

between the last quadrupole and the IP, and the final quadrupole is always vertically focusing, since inverting the polarity of the final doublet, keeping a constant demagnification ratio between the two planes, degrades the performance. Also, for the $10-\mathrm{TeV}$ case, we studied an optics where we replaced the doublets in the final transformer by triplets, since a triplet configuration would be more natural for focusing round beams. No space has been assigned yet for sweeping dipoles, which may be needed for background suppression [8] .

Figure 1 depicts the beta functions and dispersion for a conventional finalfocus system at $10 \mathrm{TeV}$. The total length of the system is about $1500 \mathrm{~m}$. Counting both sides, the final focus would occupy about $20 \%$ of the total ring circumference $(15 \mathrm{~km})$, while for the lower-energy $100-\mathrm{GeV}$ design [9] this fraction is $50 \%$. Thus, the fraction of the collider ring assigned to the final focus decreases with beam energy, and at $100 \mathrm{TeV}$ the total length of the final focus system of about $9 \mathrm{~km}$ amounts to only $9 \%$ of the collider circumference $(100 \mathrm{~km})$.

Table 2 summarizes the main parameters of six different optical models studied. Figures 2 and 3 compare the momentum acceptance for two of these optics. Table and figures demonstrate that increasing the quadrupole gradient widens the acceptance. The best performance is obtained for the highest gradients, e.g., with optics '10-TeV b' at $10 \mathrm{TeV}$ and '100-TeV c' at $100 \mathrm{TeV}$. 


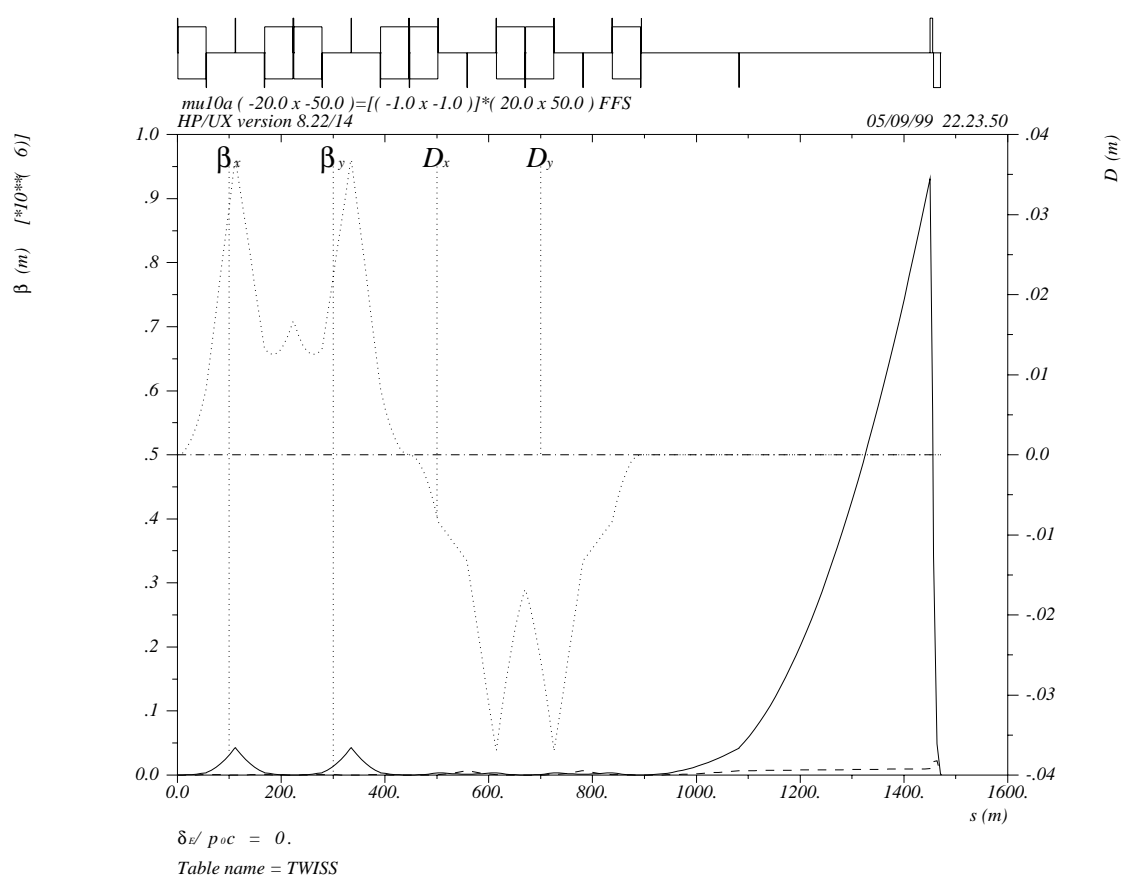

Figure 1: Twiss functions for a $10-\mathrm{TeV}$ final focus ('10-TeV a') with a maximum quadrupole gradient of $320 \mathrm{~T} / \mathrm{m}$.

All examples for $100 \mathrm{TeV}$ assume enormous final quadrupole gradients of either $3200 \mathrm{~T} / \mathrm{m}$ or $7200 \mathrm{~T} / \mathrm{m}$, without which we could not find a satisfactory optics solution. Not only at $100 \mathrm{TeV}$, but already for the $10-\mathrm{TeV}$ systems the required quadrupole gradients are incompatible with the desired beam stay clear assuming present magnet technology [10]. For example, with a peak beta function of almost $1000 \mathrm{~km}$ in optics ' $10-\mathrm{TeV}$ a' and using the beam parameters of Table 1 , the maximum rms beam size inside the last two quadrupoles is $2.8 \mathrm{~cm}$. If we require at least $2 \sigma$ beam stay clear and another $3 \mathrm{~cm}$ space for a tungsten liner, the inner radius of the final quadrupoles must be $9 \mathrm{~cm}$, which is $3-4$ times larger than the maximum aperture in present high-gradient superconducting quadrupole designs [5].

Therefore, we must assume one of three possibilities: either a breakthrough in magnet technology, or the use of novel high-gradient focusing methods, or emittances which are substantially smaller than those listed in Table 1. The first option is too speculative. Dynamic focusing or plasma lenses, which come to mind for the second option, are not well adapted to a multi-turn application. It remains the third option, namely to demand much smaller transverse emittances. Reduced emittances would also alleviate problems with higher-order optical aberrations 


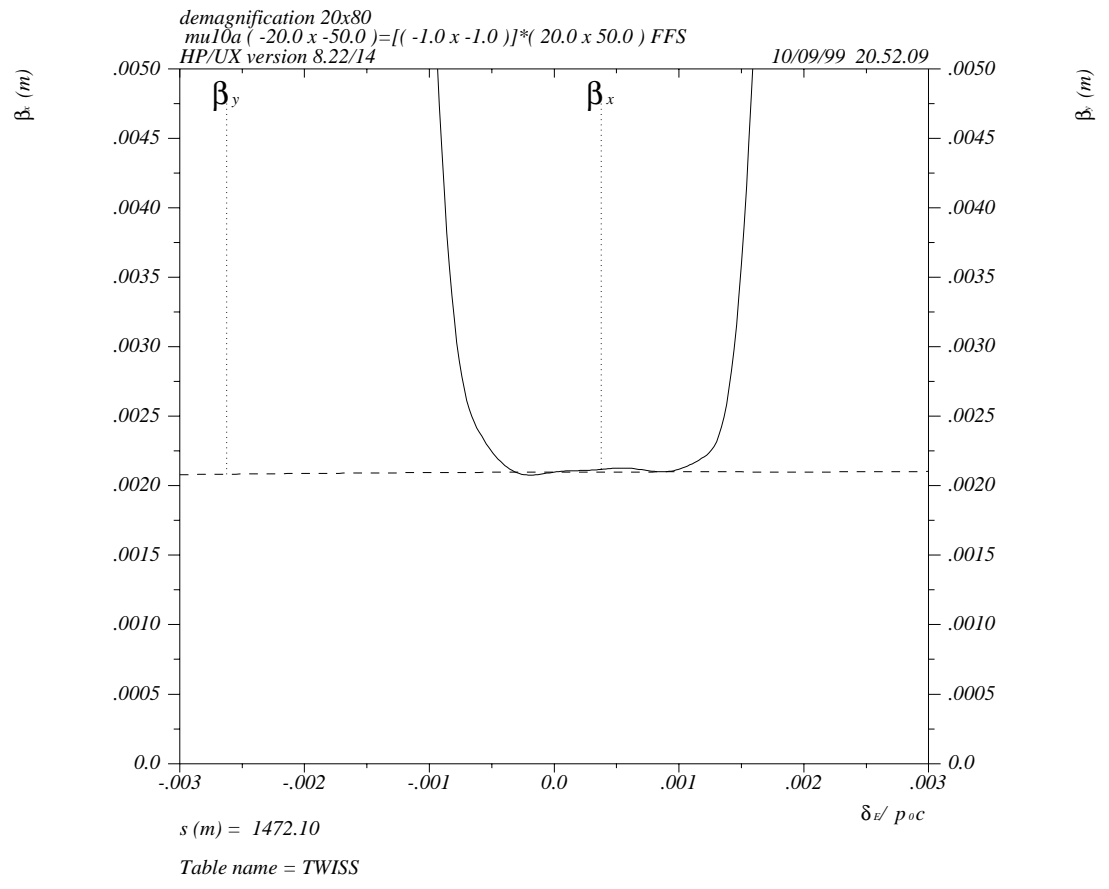

Figure 2: Momentum bandwidth for final-focus optics $10-\mathrm{TeV}$ a, with a finalquadrupole gradient of $320 \mathrm{~T} / \mathrm{m}$. Shown is the beta function as a function of the relative momentum deviation.

which are discussed next.

Although for all six test optics the bandwidth in the horizontal plane is not large, in some cases it appears barely sufficient for the extremely small energy spread of the high-energy muon beam. However, tracking shows that the situation is worse. None of the presented systems achieves a satisfactory performance in the horizontal plane, due to higher-order chromo-geometric aberrations. So the horizontal spot size for optics ' $10-\mathrm{TeV}$ b' with a $\pm 0.042 \%$ flat energy distribution is $35 \mu \mathrm{m}$, instead of the expected $1.3 \mu \mathrm{m}$.

Figure 4 shows the dependence of the horizontal blow-up on the energy spread and on the transverse emittance, demonstrating that it is caused by higher-order chromo-geometric terms. Without energy spread, the horizontal spot size shrinks to $1.78 \mu \mathrm{m}$. We have not identified the cause of the residual small blow-up for zero energy spread (imperfections of the phase advances between sextupoles are one possibility). Figure 5 illustrates that this blow up vanishes for a 10-times smaller emittance. For nominal emittances the vertical spot size is still close to the ideal value.

The chromaticity $\xi$ listed in Table 2 is defined in the linear collider sense. It quantifies the increase of the IP spot size due to an rms momentum spread $\delta_{\text {rms }}$ 


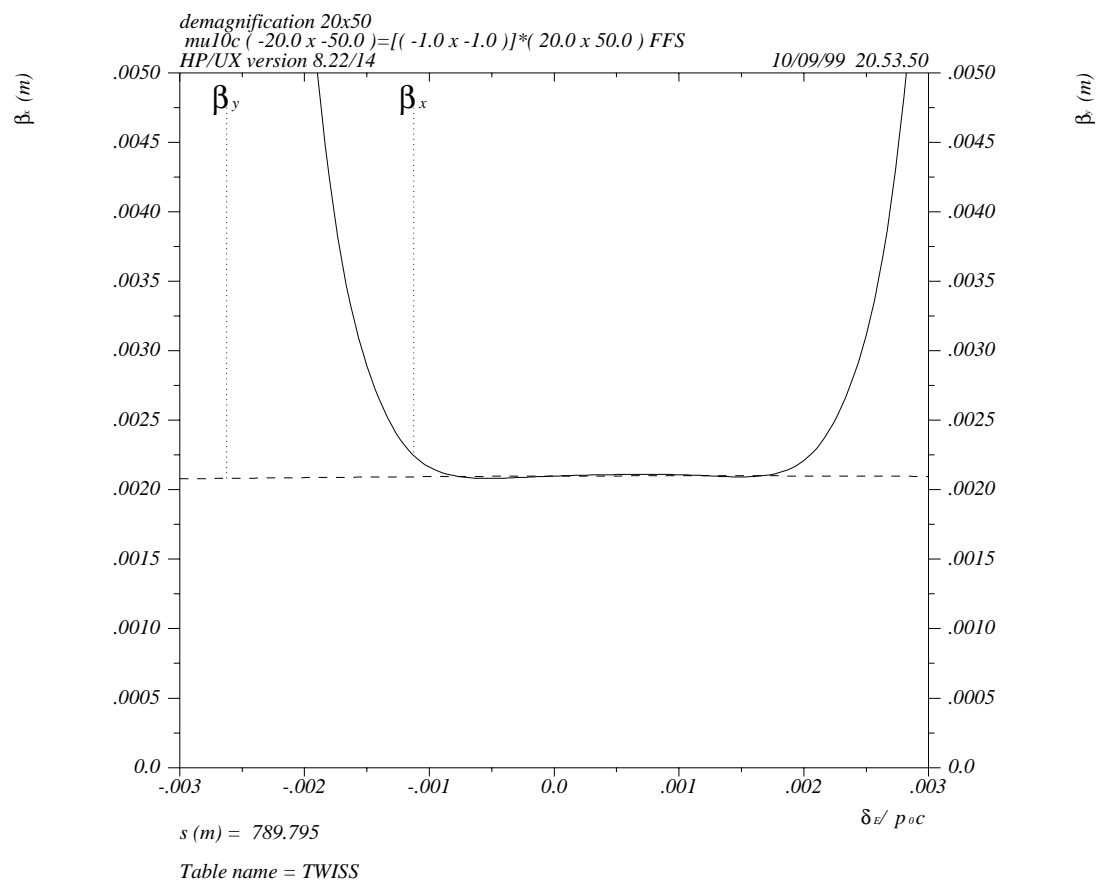

Figure 3: Momentum bandwidth for final-focus optics $10-\mathrm{TeV}$ b, with a finalquadrupole gradient of $3200 \mathrm{~T} / \mathrm{m}$. Shown is the beta function as a function of the relative momentum deviation.

according to

$$
\frac{\Delta \sigma}{\sigma_{0}}=\xi \delta_{\mathrm{rms}}
$$

The actual spot size is obtained as the quadratic sum of the linear spot size $\sigma_{0}$ and the chromatic contribution $\Delta \sigma$ :

$$
\sigma=\left(\sigma_{0}^{2}+(\Delta \sigma)^{2}\right)^{1 / 2}
$$

The sextupoles in the chromatic correction section are adjusted so that the total chromaticity $\xi$ is zero. Therefore, to first order, the incoming energy spread has no effect on the IP spot size. However, if a certain amount of energy spread $(\Delta \delta)_{\mathrm{rms}}$ is generated between the sextupoles and the final doublet, it will interact only with the uncompensated chromaticity of the final doublet and increase the spot size. Such energy spread can be generated by longitudinal wake fields, space charge, or synchrotron radiation. 




Figure 4: Simulated horizontal rms IP spot size as a function of the half width of a flat energy distribution, for optics ' $10-\mathrm{TeV}$ b'. Results are shown for the nominal and for a ten times decreased emittance. The vertical axis shows the spot size divided by its value expected from the linear optics.

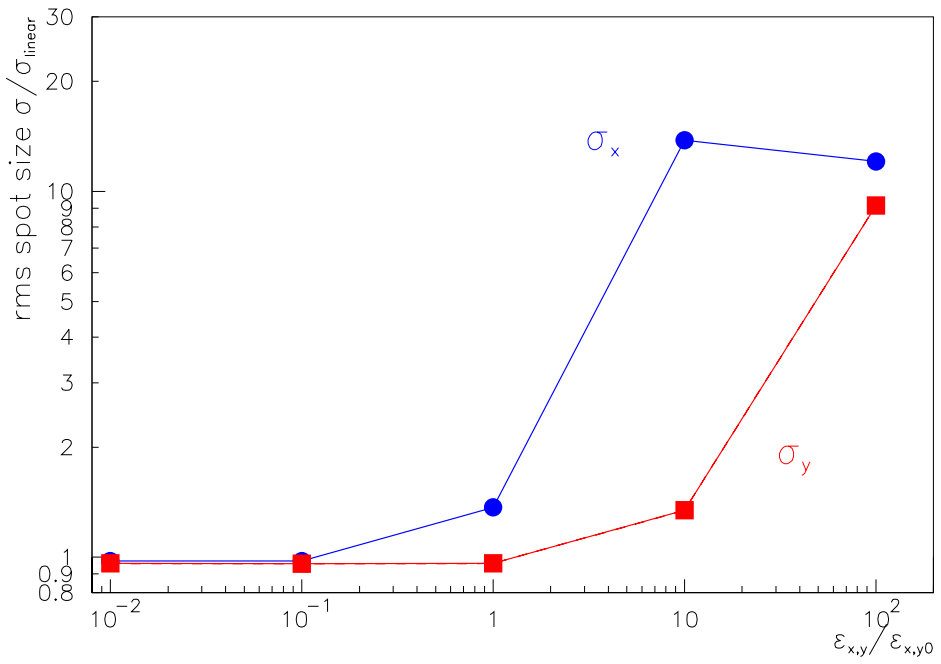

Figure 5: Simulated rms IP spot sizes with zero energy spread as a function of the transverse emittances, for optics ' $10-\mathrm{TeV}$ b'. The vertical axis shows the spot size divided by its value expected from the linear optics; depicted along the horizontal axis is the emittance normalized to the design emittance. 
Table 2: Some parameters for the different final-focus optics considered. Listed are the name of the optics, the maximum field gradient in the final quadrupoles, the horizontal and vertical chromaticities, the final-focus length per side, and the total momentum acceptance.

\begin{tabular}{lccccc}
\hline optics & $\left(\partial B_{y} / \partial x\right)_{\max }$ & $\xi_{x}$ & $\xi_{y}$ & $L$ & $(\Delta p / p)_{\mathrm{FW}}$ \\
\hline \hline $10-\mathrm{TeV} \mathrm{a}$ & $320 \mathrm{~T} / \mathrm{m}$ & 56000 & 3500 & $1472 \mathrm{~m}$ & $0.15 \%$ \\
$10-\mathrm{TeV} \mathrm{b}$ & $3200 \mathrm{~T} / \mathrm{m}$ & 17000 & 1500 & $790 \mathrm{~m}$ & $0.28 \%$ \\
$10-\mathrm{TeV} \mathrm{c}$ & $320 \mathrm{~T} / \mathrm{m}$ & 30000 & 10800 & $1660 \mathrm{~m}$ & $0.22 \%$ \\
$100-\mathrm{TeV} \mathrm{a}$ & $3200 \mathrm{~T} / \mathrm{m}$ & 39500 & 7000 & $4500 \mathrm{~m}$ & $0.14 \%$ \\
$100-\mathrm{TeV} \mathrm{b}$ & $3200 \mathrm{~T} / \mathrm{m}$ & 39500 & 7000 & $3000 \mathrm{~m}$ & $0.12 \%$ \\
$100-\mathrm{TeV} \mathrm{c}$ & $7200 \mathrm{~T} / \mathrm{m}$ & 26000 & 4700 & $4500 \mathrm{~m}$ & $0.21 \%$ \\
\hline
\end{tabular}

\section{$3 \quad$ Wake Fields and Space Charge}

The higher bunch charge, compared with most other accelerators, motivates a careful look at wake fields and space charge effects. Transverse wake fields amplify any initial orbit fluctuation, and, thereby, cause the beams to only partially overlap at the collision point, especially on later turns. Longitudinal wake fields and space charge impair the chromatic correction, if the energy spread which they induce between sextupoles and the final quadrupoles becomes comparable to the inverse chromaticity $1 / \xi_{x, y}$. If the error in the chromatic correction accumulates over $n$ turns, as it probably will, the tolerance on the induced energy spread is $1 /\left(\xi_{x, y} n\right)$. Using the parameters of Table 2 and $n=1000$ turns the tolerable energy spread induced between sextupoles and final doublet is only a few times $10^{-8}$ ! Already tiny changes in the particle energies can spoil the chromatic correction, especially if they accrue over multiple turns.

The discussion of the longitudinal wake fields follows that for the NLC [11]. At the muon collider, the bunch length is long compared to the characteristic distance $s_{0}$ defined by

$$
s_{0}=\left(\frac{c b^{2}}{2 \pi \sigma_{c}}\right)^{1 / 3} \quad(\operatorname{cgs} \text { units }),
$$

where $b$ is the chamber radius, $c$ the speed of light, and $\sigma_{c}$ the conductivity. For an aluminium chamber $\left(\sigma_{c} \approx 3.2 \times 10^{17} \mathrm{~s}^{-1}\right)$ with $b=1 \mathrm{~cm}$ radius: $s_{0}=25 \mu \mathrm{m}$.

Following Refs. $[11,12]$, the change in rms energy spread due to the resistivewall wake field reads

$$
\frac{d \sigma_{E}}{d s}=0.205 \frac{N e^{2}}{b^{2}}\left(\frac{s_{0}}{\sigma_{z}}\right)^{3 / 2} \quad \text { (cgs units). }
$$


With aluminium, and in more practical units, the induced relative energy spread is

$$
(\Delta \delta)_{\mathrm{rms}}=3.6 \frac{N\left[10^{12}\right]}{E[\mathrm{eV}] b[\mathrm{~cm}]\left(\sigma_{z}[\mathrm{~cm}]\right)^{3 / 2}} \Delta s[\mathrm{~cm}],
$$

where $\Delta s$ is the length of the beamline segment considered. For an aluminium beam pipe with $1 \mathrm{~cm}$ radius the induced energy spread is $(\Delta \delta)_{\mathrm{rms}} \approx 3 \times 10^{-8}$ at $10 \mathrm{TeV}$ assuming a length $\Delta s \approx 1.5 \mathrm{~km}$, and it is $(\Delta \delta)_{\mathrm{rms}} \approx 2 \times 10^{-9}$ at $100 \mathrm{TeV}$ over $\Delta s \approx 4.5 \mathrm{~km}$. This is not far from the tolerable limit.

To suppress the enormous background from muon decay, a large number of collimators in the final focus may be necessary [8]. Geometric wake fields from these collimators also generate an rms energy spread, and can impair the chromatic correction. For an untapered collimator of radius $a$ in a beam pipe of radius $b$ the induced rms energy spread is $[11,13,14,15]$

$$
(\Delta \delta)_{\mathrm{rms}} \approx 0.444 \times \frac{N r_{\mu}}{\gamma \sigma_{z}} \ln \frac{b}{a} .
$$

For example, consider a collimator at $1.5 \mathrm{~cm}$ in a 2 -cm beam pipe. At $10 \mathrm{TeV}$ the rms energy spread is $(\Delta \delta)_{\mathrm{rms}} \approx 7 \times 10^{-8}$, and at $100 \mathrm{TeV},(\Delta \delta)_{\mathrm{rms}} \approx 1.6 \times 10^{-9}$.

The $10-\mathrm{TeV}$ value is already significant. In addition, the contributions of different collimators to $(\Delta \delta)_{\text {rms }}$ add linearly. Therefore, if there are about 100 untapered collimators between sextupoles and final doublet, their geometric wake field can drastically degrade the chromatic correction and increase the IP spot size.

The effect can be reduced if the collimators are tapered. According to Ref. [16], the rms energy spread caused by a tapered collimator in the high-frequency limit is

$$
(\Delta \delta)_{\mathrm{rms}}=0.44 \times \frac{N r_{\mu}}{\gamma \sigma_{z}}\left(1-\tilde{\eta}_{1}\right) \ln \frac{b}{a},
$$

where $\tilde{\eta}=\min \left(1.0, \eta_{1}\right)$ with

$$
\eta_{1}=\frac{g \sigma_{z}}{(b-a)^{2}}
$$

and $g$ the length of the taper. If the taper is sufficiently shallow, then $\tilde{\eta}_{1}=1$, and in the approximation used zero additional energy spread is generated. For example, with a bunch length of $2.2 \mathrm{~mm}$ and a change in the chamber radius $(b-a)$ of $5 \mathrm{~mm}$, the condition $\tilde{\eta}_{1}=1$ is fulfilled, if the taper is slightly longer than $1 \mathrm{~cm}$.

Also the longitudinal space-charge force induces an rms energy spread that can affect the chromatic correction [17]. Ignoring the dependence on the transverse coordinates, the difference in energy spread between two locations ' 1 ' and ' 2 ' can be estimated as

$$
(\Delta \delta)_{\mathrm{rms}} \approx 0.28 \times \frac{2 N r_{\mu}}{\sqrt{2 \pi} \sigma_{z} \gamma} \ln \left(\frac{\left(\sigma_{x}+\sigma_{y}\right)_{2} b_{1}}{\left(\sigma_{x}+\sigma_{y}\right)_{1} b_{2}}\right),
$$


where $b_{1}$ and $b_{2}$ denote the beam pipe radius at the two locations. Assuming that the beam-pipe radius is the same, the change in rms energy spread between the sextupoles and the final doublet is determined by the ratio of the sum of horizontal and vertical beta functions at these two locations. If no care is taken, this can easily be a factor of 20 (see Fig. 1 ), which would amount to $(\Delta \delta)_{\mathrm{rms}} \approx 2.6 \times 10^{-7}$. This would be acceptable for a single turn, but it will degrade the chromatic correction over a few hundred turns. Detailed simulations with the exact spacecharge field could be performed, e.g., using a modified version of MAD as in Ref. [17].

We now discuss the transverse wake fields. The centroid deflection due to the resistive-wall wake field is given by [3]

$$
\Delta y^{\prime}=2 \frac{N r_{\mu}}{\gamma \sigma_{z}} \frac{L}{b^{3}}<f_{R}>\sqrt{\lambda \sigma_{z}}(\Delta y),
$$

where $(\Delta y)$ denotes the offset of the beam in the vacuum chamber, $b$ the beampipe radius, $\left\langle f_{R}>=0.82\right.$ a factor arising from averaging the wake field over the longitudinal bunch distribution, and $\lambda=0.045,0.15$, and $1.2 \mathrm{~nm}$ for $C u, W, T i$ [3]. For a tungsten beam pipe of 2-cm radius extending over a length of $100 \mathrm{~m}$, the jitter enhancement is $\Delta y / y \approx 5 \times 10^{-6} \beta[\mathrm{m}]$ for the $10-\mathrm{TeV}$ parameters, where $\beta$ denotes the beta function at the location of the wake. So, with beta functions reaching values $1000 \mathrm{~km}$ the transverse resistive-wall wake could be significant.

Finally, we estimate the transverse geometric wake field from a collimator (or mask), assuming that the bunch length is short compared with all radial apertures. If the collimator is not tapered, the centroid wake field kick is $[18,19]$

$$
\Delta y^{\prime}=\frac{2 N r_{\mu}}{\gamma}\left(\frac{1}{a^{2}}-\frac{1}{b^{2}}\right) y
$$

where $a$ and $b$ are the radius of the collimator and the beam pipe, respectively, and $y$ the centroid offset. For $10-\mathrm{TeV}$ and considering a transition between $1 \mathrm{~cm}$ and $1.5 \mathrm{~cm}$ radius, it evaluates to $\Delta y^{\prime} \approx 10^{-5} y[\mathrm{~m}]$. This is two times larger than the deflection from the resistive wall wake.

Fortunately, as in the longitudinal case, a taper can again reduce the size of the geometric wake field. With a taper of length $g$, the wake-field kick reads [3]

$$
\Delta y^{\prime}=\frac{N r_{\mu}}{\gamma \sigma_{z}} \frac{2(b-a)^{2}}{a b g}<f_{G}>y
$$

with $<f_{G}>=0.282$.

In conclusion, wake fields and space charge will impose strong constraints on optics design, beam-pipe apertures, and beam parameters.

\section{Synchrotron Radiation and Beamstrahlung}

Since muons are 200 times heavier than electrons, at $100 \mathrm{TeV}$ the synchrotron radiation in the final-focus bending magnets becomes comparable to that at a 
$500-\mathrm{GeV} \mathrm{e}^{+} \mathrm{e}^{-}$collider. The rms energy spread induced by synchrotron radiation over a bending length $l_{B}$ with a total bend angle $\theta$ is [20]

$$
\delta_{\mathrm{rms}}=\left(\frac{55}{24 \sqrt{3}} r_{\mu} \lambda_{\mu} \gamma^{5} \frac{\theta^{3}}{l_{B}^{2}}\right)^{1 / 2},
$$

where $\gamma=E_{\mu} /\left(m_{\mu} c^{2}\right)$ denotes the Lorentz factor, $r_{\mu}$ the classical muon radius, and $\lambda_{\mu}$ the muon Compton wavelength. For example, assuming a total bending angle of only $2.5 \mathrm{mrad}$ over a length of $2000 \mathrm{~m}$ at $50 \mathrm{TeV}$ beam energy, we find $\delta_{\text {rms }}=2 \times 10^{-9}$, which is insignificant, since the synchrotron radiation is incoherent and the energy spread increases as the square root of the number of turns. As the opposite extreme, suppose next that the strength of the dipole magnets is chosen equal to $10 \mathrm{~T}$, e.g., in order to optimize the momentum bandwidth. In this case, the total bending angle over a 2-km length is $118 \mathrm{mrad}$, and the rms energy spread evaluates to $\delta_{r m s} \approx 6 \times 10^{-7}$, which after 1000 turns becomes $2 \times 10^{-5}$. This is now comparable to the inverse chromaticity and will affect the IP spot size.

The synchrotron radiation in the last quadrupoles (Oide effect) is not an issue, because the IP beta function is more than 10 times larger than for electronpositron colliders. Also beamstrahlung, i.e., synchrotron radiation in the field of the opposing beam is still small. Two parameters characterizing the beamstrahlung are $\Upsilon$ and $n_{\gamma}$. Apart from a factor $2 / 3$, the $\Upsilon$ parameter is equal to the critical energy of the beamstrahlung in units of the incident beam energy. Its average value over the collision is [21]

$$
\Upsilon=\frac{5}{6} \frac{r_{\mu}^{2} \gamma N_{b}}{\alpha \sigma_{z} \sigma_{x}\left(1+\sigma_{y} / \sigma_{x}\right)},
$$

where $\alpha$ denotes the fine-structure constant, and $r_{\mu}$ the classical muon radius. For the $100-\mathrm{TeV}$ parameters (see Table 1 ) we find $\Upsilon \approx 3 \times 10^{-5}$. The number of beamstrahlung photons emitted per muon is [21]

$$
n_{\gamma} \approx \frac{2 \alpha r_{\mu} N_{b}}{\sigma_{x}\left(1+\sigma_{y} / \sigma_{x}\right)}
$$

which evaluates to $n_{\gamma} \approx 0.4$. This is not completely insignificant. Finally, the average beamstrahlung energy loss per collision reads $\delta_{B} \approx \Upsilon n_{\gamma} / 2$, and for 100 $\mathrm{TeV}$ it is $\delta_{B} \approx 7 \times 10^{-6}$. After 100 turns the average energy loss is equal to the rms energy spread.

In conclusion, at $100 \mathrm{TeV}$ synchrotron radiation and beamstrahlung start to become noticeable and their effect should be included in the final-focus design.

\section{$5 \quad$ Kinematic Terms}

The dynamic aperture of the muon collider ring may be limited by nonlinear kinematic terms [22]. The general trajectory equations in a magnetic field read 


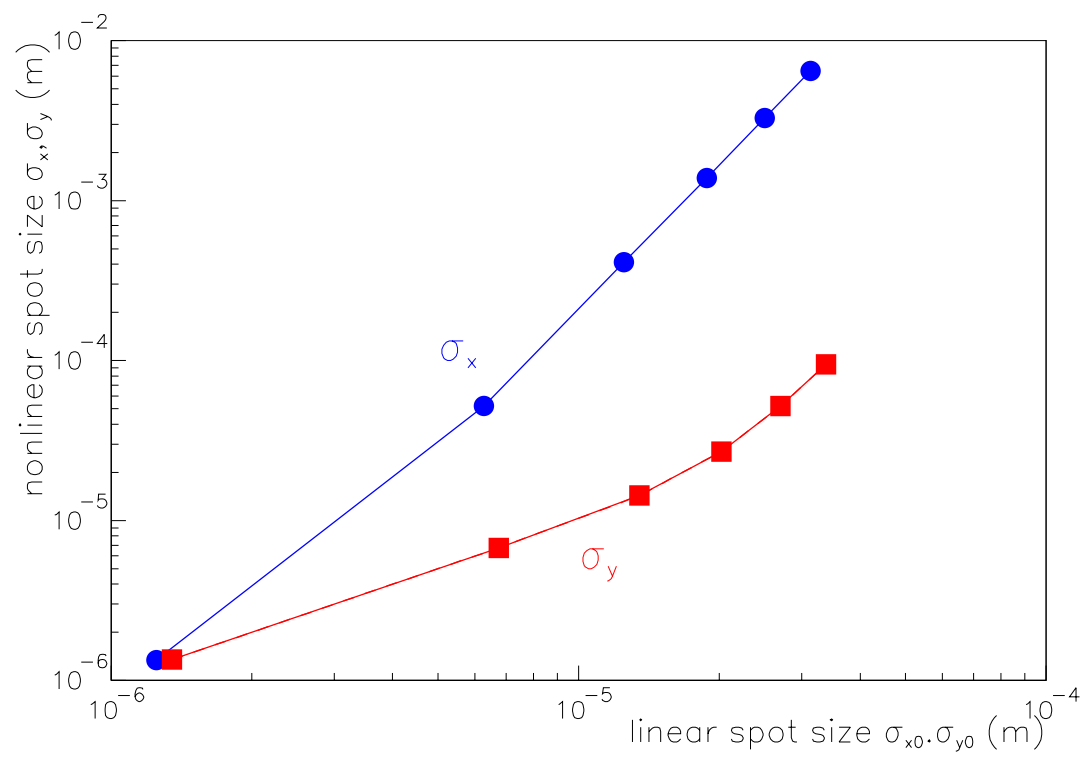

Figure 6: Spot size including kinematic terms as a function of the linear spot size, for the optics ' $10-\mathrm{TeV}$ a' with a constant $\beta_{x, y}^{*}$ and varying emittance.

[23]:

$$
\begin{aligned}
& x^{\prime \prime}=\frac{e}{p} \sqrt{1+{z^{\prime}}^{2}+{x^{\prime}}^{2}}\left[x^{\prime} B_{s}-\left(1+z^{\prime 2}\right) B_{x}+x^{\prime} z^{\prime} B_{z}\right] \\
& z^{\prime \prime}=-\frac{e}{p} \sqrt{1+z^{\prime 2}+x^{\prime 2}}\left[z^{\prime} B_{s}-\left(1+z^{\prime 2}\right) B_{z}+x^{\prime} z^{\prime} B_{x}\right]
\end{aligned}
$$

with $p=\gamma m v$ the particle momentum.

To examine the importance of this effect, we have tracked a distribution of 100 on-momentum particles through the final focus. We varied the initial emittances, and performed simulations with and without the nonlinear kinematic terms. Results are illustrated in Fig. 6. For the nominal emittance at $10 \mathrm{TeV}$, the effect of the nonlinear contributions is small. They increase the rms spot size by less than $1 \%$. However, if we track a distribution with a 25 times larger emittance (in both planes) the rms spot size at the IP is ten times larger with kinematic terms than without. This suggests that the kinematic terms may indeed affect the dynamic aperture.

At $100 \mathrm{TeV}$, for optics '100-TeV a', the same relative blow up by a factor 10 is obtained for a 250-fold increase in the nominal emittance. Thus, the kinematic terms appear to lose importance towards higher energies. 

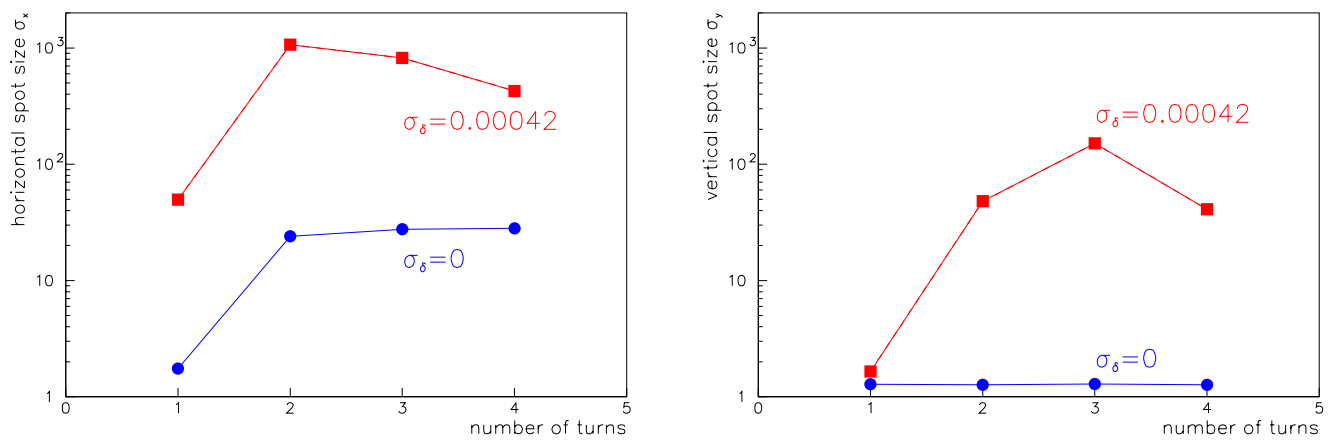

Figure 7: Simulated rms IP spot sizes as a function of turn number, with and without beam energy spread, for optics ' $10-\mathrm{TeV}$ b' and choosing a fractional tune of 0.31 in both transverse planes.

\section{Multi-Turn Effects}

Table 3 lists some general muon-collider parameters at 10 and $100 \mathrm{TeV}$. At $10 \mathrm{TeV}$ the muons are stored in the collider ring for 740 turns; at $100 \mathrm{TeV}$ they are stored for 380 turns. At the end of the $10-\mathrm{TeV}$ store the luminosity has decreased by $50 \%$, and the two beam currents by about 30\%. Optimum luminosity is achieved if new bunches are injected into empty rf buckets, while the old bunches continue to collide until no particles are left.

Table 3: A few collider parameters at 10 and $100 \mathrm{TeV}$, as provided by the workshop organizers.

\begin{tabular}{lcc}
\hline energy & $10 \mathrm{TeV}$ & $100 \mathrm{TeV}$ \\
circumference & $15 \mathrm{~km}$ & $100 \mathrm{~km}$ \\
repetition rate & $27 \mathrm{~Hz}$ & $7.9 \mathrm{~Hz}$ \\
\hline
\end{tabular}

Even if the design IP spot size is reached on the first turn, it will be difficult to maintain this spot size on subsequent turns. Figure 7 shows a preliminary simulation result for optics ' $10-\mathrm{TeV}$ b', which illustrates a significant blow-up on later turns. The horizontal blow-up for zero energy spread could have its origin in imperfections of the $-I$ or of the phase advance between the sextupoles and the final doublet, and/or in a small mismatch between turns. A larger concern is the enormous spot-size increase for a nonzero (but small) energy spread, which we attribute to higher-order chromogeometric aberrations. 

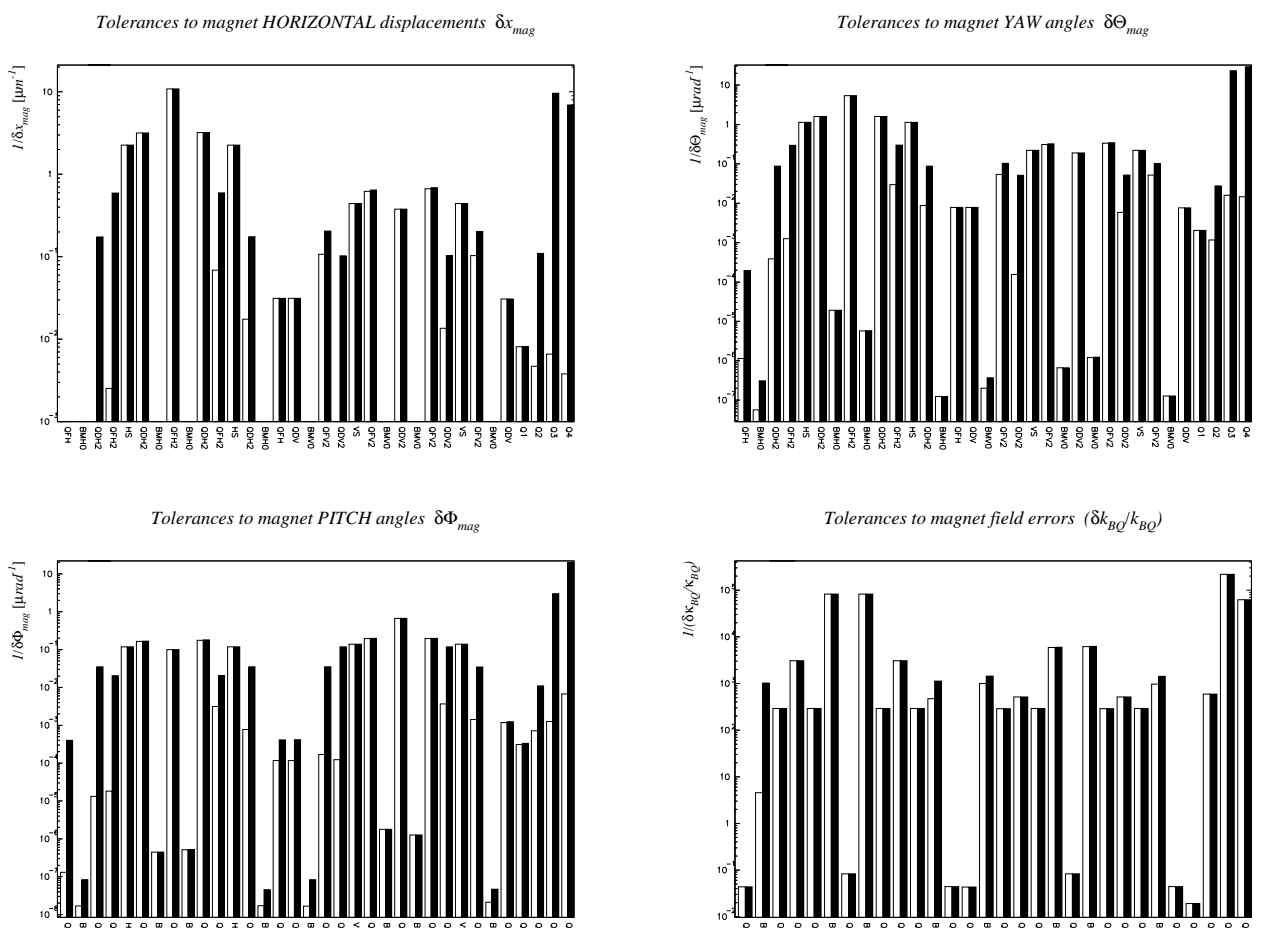

Figure 8: Single-pass tolerances for quadrupole, bending and sextupole magnets in optics '10-TeV a'. Shown are tolerances on magnet position, yaw angle, pitch angle, and field strength. Each value displayed corresponds to a luminosity loss of $2 \%$. The full bars represent pulse-to-pulse 'jitter' tolerances, due to the induced orbit motion at the interaction point. This jitter can be corrected within a few pulses using a fast orbit feedback. The open bars are 'drift' tolerances referrring to increases in the IP spot size. Since the beam size tuning will be performed less frequently, these tolerances must be met over a longer time scale, e.g., minutes.

\section{Tolerances and Tuning}

Single-pass tolerances can be calculated with the FFADA code [6]. Figure 8 shows tolerances on transverse magnet position, magnet yaw and pitch angles, and quadrupole field strengths, for the optics ' $10-\mathrm{TeV}$ a'. According to the calculations, the final quadrupoles must be stabilized at the 20-nm level. Without feedback the multi-turn tolerances are significantly tighter than the single-pass tolerances. Thus, a turn-by-turn orbit feedback may be indispensible.

At the SLC, the different aberrations, such as dispersion, waist, and linear coupling, were corrected at regular time intervals. These corrections were performed by scanning and adjusting combinations of quadrupole and skew quadrupole magnets (so-called multiknobs), such that the IP spot sizes were minimized or the 

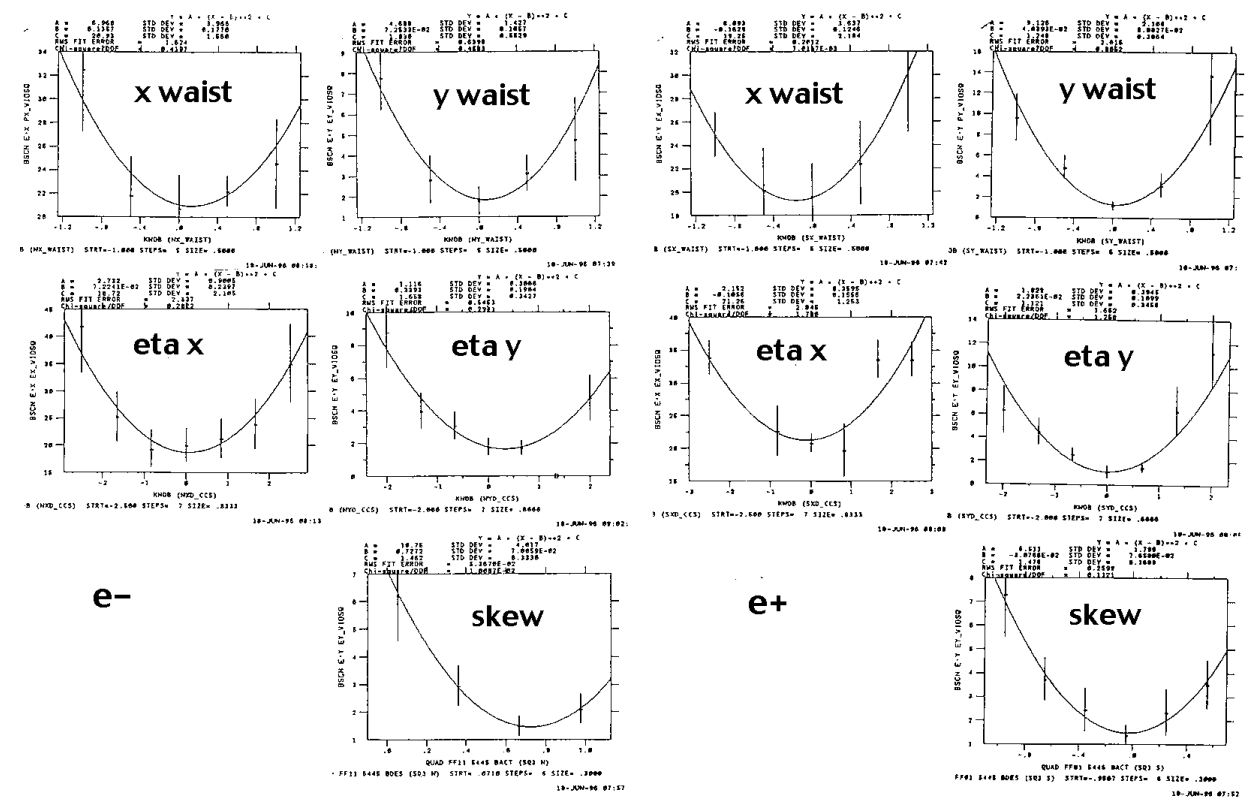

Figure 9: Typical tuning scans at the SLC interaction point. Dispersion, waist and coupling were corrected evey few hours. To this end, orthogonal multiknobs were varied, and for each multiknob setting the rms beam sizes determined via beam-beam deflection scans. The knobs were set to the minimum of the fitted parabola.

luminosity maximized. The spot sizes were determined from beam-beam deflection scans; luminosity and beamstrahlung could be measured with dedicated monitors.

At a muon collider, both beams would be affected by changes in the quadrupole settings, which will complicate the tuning procedures, unless only beam observations from the first turn are used. For independent control of the two beams, it may be necessary to use electrostatic quadrupole magnets, a challenge at these beam energies. Moreover, the multiknobs should not change any of the betatron tunes. There may also be a need or desire to match the optics from turn to turn, which perhaps could be done with pulsed quadrupole magnets. Different from a single pass collider, also the outgoing aberrations must be matched, which doubles the number of tuning scans required.

Tuning will thus become more difficult. However, the many turns also open up, in principle, the possibility to perform several complete tuning scans during one cycle. 


\section{Background, Heat Load and Collimation Issues}

There are two sources of background: (1) muon decay [8], and (2) beam halo [24]. Only the second source exists in electron-positron colliders. Over a distance of 1 $\mathrm{km} 10^{8}$ muons are lost at $10 \mathrm{TeV}$, and $2 \times 10^{6}$ at $100 \mathrm{TeV}$.

As a means to reduce the background and the heat load of superconducting quadrupoles due to the decaying muons, it was proposed to install sweeping magnets. For a $2-\mathrm{TeV}$ collider, these sweeping dipoles cover about $40 \%$ of the final-focus length and have a strength of $8.5 \mathrm{~T}$. There may even be a need for sweeping magnets between the IP and the first (last) quadrupoles, which might lengthen $l^{*}$ and enhance the chromaticity of the system.

A second approach, for reduced heat load, is to add a several $\mathrm{cm}$ thick tungsten liner inside the quadrupoles. This of course lowers the quadrupole gradient and, like the sweeping dipoles, it will further aggravate the problem of higher order chromaticity.

\section{Single-Pass Muon Collider}

The design of a muon ring collider at multi- $\mathrm{TeV}$ energies faces severe, perhaps insurmountable problems:

- The neutrino radiation is likely to limit a ring collider to energies below a few $\mathrm{TeV}$. The radiation hazard arises because the neutrino cross sections increase almost linearly with energy, while the angular divergence of the emitted neutrinos decreases as $1 / \gamma$. As a net result the neutrino radiation dose increases as the 3rd power of energy [25], and at multi-TeV energies easily exceeds the US Federal limit [26].

- The beam has to survive hundreds of passes through a final-focus system more challenging than that of the SLC, retaining the same constant emittance. This appears non-trivial, as already the extracted beam at the SLC showed large emittance degradation even in the absence of collisions.

- If optical stochastic cooling [27, 28, 29], or other future techniques reduce the muon beam emittances by several orders of magnitude, the luminosity of a ring collider is limited by the beam-beam tune shift.

- Synchrotron radiation in the collider ring will likely increase the nominal beam emittance [30], casting doubt on the value of ultracold muon beams for high-energy muon ring colliders.

- Conventional quadrupole magnets appear to be too weak for a satisfactory optics design; advanced high-gradient schemes like plasma lenses or dynamic focusing are not well suited for a multi-pass system.

Similarly, several difficulties lie in the way of an electron-positron linear collider at multi-TeV energies. The most dramatic is the effect of beamstrahlung, 
the coherent pair creation at high $\Upsilon$, and the associated degradation of the luminosity spectrum and large background.

A single-pass muon collider (SPMC) solves all the above problems: Because of the larger muon mass, the beamstrahlung at $10 \mathrm{TeV}$ or $100 \mathrm{TeV}$ is still contained. There is no need to preserve the emittances after the collision, and the beam can be dispersed onto a dump (downwards, or upwards), thereby reducing the density of neutrino radiation by orders of magnitude. Note that, as an option, the beams could still be accelerated in a ring [31], from which they might then be extracted, focused to a small spot size, and collided only once.

Table 4: Example parameters for single-pass muon colliders at 10, 100 and 1000 $\mathrm{TeV}$.

\begin{tabular}{|c|c|c|c|c|c|}
\hline parameter & symbol & SPMC-0 & SPMC-I & SPMC-II & SPMC-III \\
\hline cm energy [TeV] & 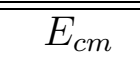 & 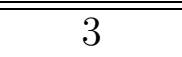 & $\overline{10}$ & 100 & 1000 \\
\hline luminosity $\left[10^{35} \mathrm{~cm}^{-2} \mathrm{~s}^{-1}\right]$ & $L$ & 1.2 & 2.1 & 7.2 & 5.4 \\
\hline beam energy $[\mathrm{TeV}]$ & $E_{b}$ & 1.5 & 5 & 50 & 500 \\
\hline muons/bunch $\left[10^{12}\right]$ & $N_{b}$ & 5 & 3 & 0.8 & 0.2 \\
\hline bunches/train & $n_{b}$ & 1 & 1 & 1 & 1 \\
\hline repetition rate $[\mathrm{Hz}]$ & $f_{\text {rep }}$ & 160 & 27 & 7.9 & 3.2 \\
\hline normalized tr. emittances $[\mu \mathrm{m}]$ & $\gamma \epsilon_{x, y}$ & 15 & 2 & 0.5 & 0.25 \\
\hline $\begin{array}{l}\text { 6-dim. normalized emittance } \\
{\left[10^{-12} \mathrm{~m}^{3}\right]}\end{array}$ & $\gamma^{3} \epsilon_{6 d}$ & 16 & 1.5 & 0.23 & 0.30 \\
\hline rms energy spread & $\delta_{\mathrm{rms}}$ & $1 \%$ & $1 \%$ & $1 \%$ & $1 \%$ \\
\hline rms bunch length $[\mathrm{mm}]$ & $\sigma_{z}$ & 0.5 & 0.8 & 0.2 & 0.1 \\
\hline relativistic Lorentz factor $\left[10^{4}\right]$ & $\gamma$ & 1.41 & 4.7 & 47 & 473 \\
\hline IP beta functions $[\mathrm{mm}]$ & $\beta_{x, y}^{*}$ & 0.5 & 0.8 & 0.2 & 0.1 \\
\hline IP spot sizes $[\mathrm{nm}]$ & $\sigma_{x, y}$ & 730 & 184 & 14.5 & 2.3 \\
\hline beamstrahlung energy loss & $\delta_{B}$ & $7 \times 10^{-7}$ & $8 \times 10^{-6}$ & $4 \times 10^{-3}$ & 0.14 \\
\hline Upsilon parameter & $\Upsilon$ & $2 \times 10^{-6}$ & $1.0 \times 10^{-5}$ & $1.4 \times 10^{-3}$ & 0.04 \\
\hline beamstrahlung photons/lepton & $N_{\gamma}$ & 0.71 & 1.67 & 5.61 & 8.43 \\
\hline luminosity enhancement factor & $H_{D}$ & 2.00 & 3.67 & 3.77 & 2.83 \\
\hline
\end{tabular}

Table 4 shows example parameters for single-pass muon colliders at 3, 10, 100 and $1000 \mathrm{TeV}$, with typical luminosities of a few $10^{35} \mathrm{~cm}^{-2} \mathrm{~s}^{-1}$. The 6 -dimensional emittance for $3 \mathrm{TeV}$ is a factor 5 smaller than the workshop strawman-design value in Table 1. At higher energies the 6-dimensional emittance is much further reduced. For these beam energies the small IP beta functions cannot be achieved with conventional magnets, but must be based on more exotic techniques, such as a plasma lens $[32,33]$ or dynamic focusing $[34,35,36]$. The IP beta functions listed in the table correspond to the minimum values achievable with a plasma 
lens, as estimated in Eq. (20) below. The average muon current at $1000 \mathrm{TeV}$ is chosen a factor 10 smaller than at $100 \mathrm{TeV}$, in order to account for the muon decay and to limit the beam power. The $3-\mathrm{TeV}$ case is special since here conventional quadrupoles can still be used to focus the beams to the desired spot sizes. The $3-\mathrm{TeV}$ IP beta functions of $0.5 \mathrm{~mm}$ assumed in both planes are similar to those proposed for a $500-\mathrm{GeV} \gamma \gamma$ collider [37]. Since at the $3-\mathrm{TeV}$ muon collider beamstrahlung is insignificant, it will provide a much purer luminosity spectrum than a multi- $\mathrm{TeV}$ electron-positron collider.

To focus 50-TeV muon beams, advanced focusing techniques are indispensible. One option is a plasma lens. We denote the beam size at the plasma lens by $\sigma_{r}$, the beta function at the lens by $\beta_{r}$, the rms transverse beam emittance by $\epsilon$, the plasma density by $n_{p}$ and the distance between plasma lens and collision point by $l^{*}$. If the plasma density is smaller than the beam density, the focusing strength of the plasma lens is [33]

$$
K=\frac{2 \pi r_{\mu}}{\gamma} n_{p}
$$

and the reduction of the focusing field by the plasma return current is small as long as [33]

$$
k_{p} \sigma_{r} \gg 1
$$

where $k_{p}=\sqrt{4 \pi r_{e} n_{p}}$ is the plasma wave number. Combining Eqs. (18) and (19) and using the approximation $l^{*} \approx 1 / K^{1 / 2}$ we obtain a lower bound on the IP beta function achievable with a plasma lens:

$$
\beta^{*} \geq 2 \frac{r_{e}}{r_{\mu}}\left(\gamma \epsilon_{x, y}\right) .
$$

This bound depends only on the mass ratio of electrons and muons and on the normalized beam emittance. Emittances and beta functions in Table 4 were chosen in accordance with Eq. (20). If the normalized emittance can be reduced further, e.g., via optical stochastic cooling [29], the IP beta function may be decreased in proportion! The luminosity scales as

$$
L \propto \frac{1}{\left(\gamma \epsilon_{x}\right)\left(\gamma \epsilon_{y}\right)} .
$$

Another technique that has been proposed for electron-positron colliders is dynamic focusing $[34,35,36]$. For example, with a demagnification factor $\xi=$ $l^{*} / \beta^{*}$ of 33 the focusing by the lens beam would reduce the betafunction at the interaction point from about $2 \mathrm{~cm}$ to $20 \mu \mathrm{m}$. The required lens-beam charge is [35]

$$
N_{q} \approx \frac{3 \gamma \epsilon_{x, y}}{r_{\mu}} \xi,
$$

which amounts to $N_{q} \approx 2.7 \times 10^{14}$ for a muon collider at $10 \mathrm{TeV}$, and $N_{q} \approx 6 \times 10^{13}$ at $100 \mathrm{TeV}$. Due to its dependence on normalized emittance and particle mass, 
this charge is much higher than it would be for an electron beam. Also dynamic focusing becomes easier for reduced beam emittances.

\section{Conclusions}

Already for a single pass, the design of a final-focus optics for a multi-TeV muon collider is nontrivial, due to the limited strength of conventional quadrupoles and the large geometric emittances. The optics design must rely on novel focusing methods, better cooling of the muon beam, or advances in the design and construction of high-field magnets. The multiple passes imply many additional challenges concerning spot-size stability, tolerances, and tuning. A single-pass collider is a promising option which avoids these last (and many other) problems, is much better adapted to advanced focusing techniques, and could achieve a luminosity of several $10^{35} \mathrm{~cm}^{-2} \mathrm{~s}^{-1}$, with normalized emittances $\gamma \epsilon_{x, y}=2 \mu \mathrm{m}$ at $10 \mathrm{TeV}$, and $0.5 \mu \mathrm{m}$ at $100 \mathrm{TeV}$. The assumed 6-dimensional emittances are still many orders of magnitude larger than the ultimate emittance that might be attained by optical stochastic cooling [29], leaving room for substantially higher luminosity. If plasma lenses are employed, the luminosity scales inversely with the product of the normalized transverse emittances.

\section{Acknowledgements}

I am grateful to B. King for inviting me to the workshop and for continuous encouragement. I thank C. Johnstone, E. Keil and V. Telnov for helpful discussions and informations. Support by K. Hübner, J. Gareyte, and F. Ruggiero is appreciated.

\section{References}

[1] B. King, "Studies for Muon Collider Parameters at Center-of-Mass Energies of $10 \mathrm{TeV}$ and $100 \mathrm{TeV}$ ", presented at IEEE PAC99, New York (1999).

[2] SLAC Linear Collider Conceptual Design Report, SLAC-R-0229 (1980).

[3] The NLC Design Group, "Zeroth Order Design Report for the Next Linear Collider", presented at the 1996 DPF/DPB Summer Study on New Directions in High Energy Physics (Snowmass '96).

[4] J.P. Delahaye and I. Wilson for the CLIC Study Team, "CLIC, a Multi-TeV $\mathrm{e}^{+} \mathrm{e}^{-}$Linear Collider", CERN/PS 99-062.

[5] S. Caspi, K. Chow, A.F. Lietzke, A.D. McInturff, M. Morrison, R.M. Scanlan, G. Ambrosio, G. Bellomo, F. Broggi, L. Rossi, "Design of a $\mathrm{Nb}_{3} \mathrm{Sn}$ High Gradient Low-Beta Quadrupole Magnet" 15th International Conference on Magnet Technology, Bejing, China (1997). 
[6] O. Napoly and B. Dunham, "FFADA: Computer design of final focus systems for linear colliders," Contributed to 4th European Particle Accelerator Conference (EPAC 94), London, England, 27 Jun - 1 Jul 1994.

[7] H. Grote, F.C. Iselin, "The MAD Program", CERN/SL/90/13 (1990).

[8] C. Johnstone and N.V. Mokhov, "Optimization of a Muon Collider Interaction Region with Respect to Detector Backgrounds and the Heat Load to the Cryogenic Systems", presented at the 1996 DPF/DPB Summer Study on New Directions in High Energy Physics (Snowmass '96).

[9] C.M. Ankenbrandt et al., "Status of Muon Collider Research and Development and Future Plans", PRST-AB 2, no. 8., (1999).

[10] E. Keil, presentation at this workshop and private communication (1999).

[11] T. Raubenheimer and F. Zimmermann, "Longitudinal Wake Fields and Chromatic Spot-Size Dilution in the NLC Final Focus", SLAC NLC Note 23 (1996).

[12] A. Piwinski, DESY Report 72/72 (1972).

[13] V.E. Balakin and A.V. Novokhatsky, Proc. 12-th Int. Conf. High Energy Accel., Batavia, p. 117 (1984).

[14] L. Palumbo, Part. Acc. 25, pp. 201-216 (1990).

[15] S.A. Kheifets, IEEE Trans. Mcrowave Theory Technique MTT-35, 753-760 (1987).

[16] S.A. Heifets and S.A. Kheifets, "Coupling Impedance in Modern Accelerators", Rev. Mod. Physics, Vol. 63, no. 3 (1991).

[17] F. Zimmermann and T. Raubenheimer, "Longitudinal Space Charge in Final-Focus Systems for Linear Colliders", NIM A 390, p. 279 (1997).

[18] E. Gianfelice, L. Palumbo, IEEE Tr-NS, 37, 2, p. 1084 (1990).

[19] F. Zimmermann, K.L.F. Bane, C.K. Ng, "Collimator Wake Fields in the SLC Final Focus", Proc. of EPAC96, Sitges, p. 504 (1996).

[20] Sands, M., 1979, "The Physics of Electron Storage Rings", SLAC-121.

[21] Chen, P., and K. Yokoya, 1992, "Beam-Beam Phenomena in Linear Colliders", in 'Frontiers of Particle Beams: Intensity Limitations', Lecture Notes in Physics 400, Springer-Verlag.

[22] W. Wan, "Tune-Shift with Amplitude due to Nonlinear Kinematic Effect", 1999 IEEE PAC, New York (1999).

[23] K. Steffen, "High-Energy Beam Optics", Interscience Publishers (1965).

[24] A. Drozhdin, N. Mokhov, C. Johnstone, W. Wan, and A. Garren, "Scraping Beam Halo in $\mu^{+} \mu^{-}$Colliders", 4th International Conference on Physics Potential and Development of Muon Colliders", San Francisco, California (1997). 
[25] B. King, "Potential Hazards from Neutrino Radiation at Muon Colliders", presented at IEEE PAC99, New York (1999).

[26] B. King, "Neutrino Physics at a Muon Collider", Proc. of the Workshop on Physics at the First Muon Collider and Front End of a Muon Collider, Fermilab (1997).

[27] A. Mikhalichenko, M. Zolotorev, "Optical Stochastic Cooling", Phys. Rev. Lett. 71, p. 4146 (1993).

[28] M.S. Zolotorev and A.A. Zholents, "Transit time method of optical stochastic cooling", Phys. Rev. E50, 3087 (1994).

[29] A. Zholents, contribution to this workshop.

[30] V. Telnov, contribution to this workshop.

[31] C. Johnstone, remark at this workshop.

[32] P. Chen, "A Possible Final Focusing Mechanism for Linear Colliders", Part. Acc. 20, 171 (1987).

[33] W. Barletta et al., "Plasma Lens Experiments at the Final Focus Test Beam", Proc. of the 6th workshop on Advanced Accelerator Concepts, Lake Geneva (1994).

[34] J. Irwin, "Dynamic Focusing Schemes for Linear Colliders", Proc. IEEE PAC97, Vancouver, B.C., (1997).

[35] J. Irwin, R. Helm, K. Thompson, D. Schulte, "Further Developments in Dynamic Focusing", Proc. IEEE PAC99, New York (1999)

[36] J. Irwin, contribution to this workshop.

[37] C. Adolphsen et al., "Zeroth Order Design Report for the Next Linear Collider", LBNL-PUB-5424, SLAC Report 474, submitted to Snowmass 96 (1996). 